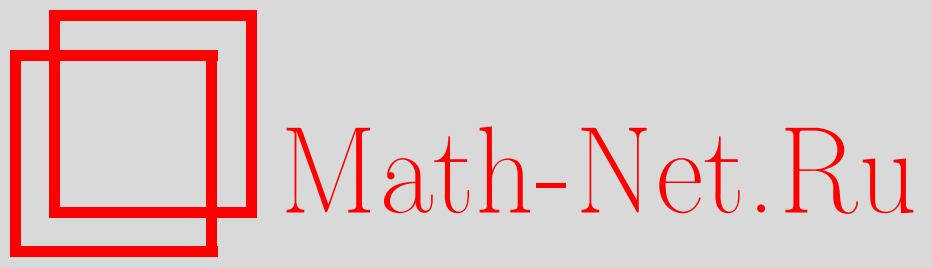

А. Л. Якымив, Об асимптотике плотности безгранично делимого распределения на бесконечности, Теория вероятн. и ее примен., 2002, том 47, выпуск 1, 80-89

DOI: https://doi.org/10.4213/tvp2995

Использование Общероссийского математического портала MathNet.Ru подразумевает, что вы прочитали и согласны с пользовательским соглашением

http://www . mathnet.ru/rus/agreement

Параметры загрузки:

IP : 3.89.185.249

26 апреля 2023 г., 16:50:07

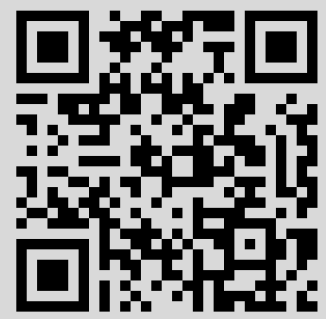




\section{ОБ АСИМПТОТИКЕ ПЛОТНОСТИ БЕЗГРАНИЧНО ДЕЛИМОГО РАСПРЕДЕЛЕНИЯ НА БЕСКОНЕЧНОСТИ ${ }^{1)}$}

В случае, когда плотность абсолютно непрерывной части меры Леви безгранично делимого распределения мажорируемо меняется на бесконечности, в статье изучены асимптотические свойства на бесконечности плотности этого распределения. Изложение ведется в терминах так называемой слабой эквивалентности функций, которая в случае слабо осциллирующей и, в частности, правильно меняющейся на бесконечности плотности безгранично делимого распределения совпадает с обычной эквивалентностью.

Ключевые слова и фразы: безгранично делимые распределения, спектральная мера Леви, плотность распределения, слабая эквивалентность функций, правильно меняющиеся функции, слабо осциллирующие функции, мажорируемо меняющиеся функции.

Асимптотика безгранично делимых распределений на бесконечности изучалась ранее в ряде работ [1]-[8], см. также ссылки в статье [1].

Хотя аналитическое строение безгранично делимых распределений было изучено еще в 1963 г. (см. [9]), асимптотика их плотности на бесконечности впервые была исследована лишь в 1990 г. [10].

В настоящей статье обобщается основной результат [10] в рассмотренном там случае неограниченной в окрестности нуля спектральной меры Леви безгранично делимого распределения. Исследован также и случай ограниченной меры Леви.

Основной результат в [10] был получен как следствие соответствующей интегральной предельной теоремы; здесь же доказательство опирается непосредственно на тауберову теорему, приводимую ниже.

Перейдем к формулировкам полученных результатов.

Пусть случайная величина $\xi$ имеет безгранично делимое распределение, т.е. для характеристической функции $\xi$ справедливо представление:

$$
\mathbf{E} e^{i t \xi}=\exp \left(i \gamma t-\frac{\sigma^{2} t^{2}}{2}+\int_{-\infty}^{+\infty}\left(e^{i t x}-1-\frac{i t x}{1+x^{2}}\right) G(d x)\right)
$$

\footnotetext{
*Математический институт им. В. А. Стеклова РАН, ул. Губкина, 8, 117966 Москва, ГСП-1, Россия. e-mail: arsen@mi.ras.ru

1) Работа выполнена при финансовой поддержке Российского фонда фундаментальных исследований (гранты № 00-15-96136, 00-01-00090).
} 
где $G(d x)$ - ее спектральная мера Леви на $(-\infty, 0) \cup(0, \infty)$ (возможно, неограниченная в окрестности нуля), причем конечны интегралы

$$
\int_{-1}^{1} x^{2} G(d x), \quad \int_{-\infty}^{-1} G(d x), \quad \int_{1}^{\infty} G(d x)
$$

$\sigma \geqslant 0, \gamma$ - некоторое действительное число, переменная $t \in(-\infty, \infty)$. Через $F(x)$ обозначим функцию распределения $\xi: F(x)=\mathbf{P}\{\xi \leqslant x\}$.

О п р е д е л е н и е 1. Для двух функций $a(t)$ и $b(t)$, заданных и неотрицательных при $t \geqslant 0$, мы будем говорить, что $a(t)$ слабо эквивалентна $b(t)$ при $t \rightarrow \infty$, и писать $a(t) \stackrel{\text { сл. }}{\sim} b(t)(t \rightarrow \infty)$, если для произвольных $\varepsilon, \delta \in(0,1)$ найдется такое $t_{0}$, что при $t \geqslant t_{0}$ выполнены неравенства

$$
(1-\varepsilon) b(t(1+\delta)) \leqslant a(t) \leqslant(1+\varepsilon) b(t(1-\delta)) .
$$

О п р е д е л е н и е 2. Фунция $a(t)$, заданная и неотрицательная при $t \geqslant 0$, называется слабо осциллирующей на бесконечности, если $a(\tau) / a(t) \rightarrow 1$ при $t \rightarrow \infty, \tau \geqslant t, \tau=t+o(t)$.

3 а м е ч а н и е 1 . Если одна из функций $a(t)$ или $b(t)$ слабо осциллирует на бесконечности, то из их слабой эквивалентности на бесконечности следует их обычная эквивалентность, т.е. $a(t) / b(t) \rightarrow 1(t \rightarrow \infty)$.

Здесь получены следующие две предельные теоремы.

Теорема 1. Пусть мера $G(d x)$ ограничена и обладает непрерьвной на $[0, \infty)$ плотностью $g(x)$, существует $\alpha>0$ такое, ито функиия $b(t)=t g(t)$ не возрастает $п р и t \geqslant \alpha$,

$$
\varlimsup_{t \rightarrow \infty} \frac{g(t)}{g(2 t)}<\infty,
$$

т.е. $g(t)$ мажорируемо мендется на бесконечности (см. [13, с. 94]), и для произвольного $\lambda>1$

$$
\varlimsup_{t \rightarrow \infty} \frac{r(\lambda t)}{r(t)}<1,
$$

аде $r(t)=\int_{t}^{\infty} g(y) d y, t>0$. Tогда при $t \rightarrow \infty$

$$
f(t) \stackrel{\text { cл. }}{\sim} g(t)
$$

əде $f(t)=d F(t) / d t, t>0$.

Теорема 2. Представим меру $G$ в виде $G=G_{A}+G_{B}$, где $G_{A}$ ее абсолютно непрерьвная часть, и пусть $g(t)-$ плотность меры $G_{A}$. Предположим, что для всех $n \in \mathbf{N}$

$$
\int_{1}^{\infty} t^{n} G_{B}(d t)<\infty
$$


существуют $\varepsilon>0 u \alpha>\varepsilon$ такие, что

$$
g(t) \geqslant t^{-1} \quad \forall t \in(0, \varepsilon],
$$

$\oint$ ункия $b(t)=t g(t)$ монотонна $и$ непрерьвна при $t \geqslant \alpha$, выполнень соотношения (1) и (2). Тогда справедливо (3).

Теорема 2 обобщает основной результат работы [10] (в [10] предполагалось, что функция $g(t)$ слабо осциллирует на бесконечности).

Следствие. Пусть мера $G(d t)$ обладает непрерывной на $[0, \infty)$ плотностью $g(t)$, представимой в виде $g(t)=t^{-\beta} l(t), \beta>1$, где функиия $l(t)$ медленно меняется на бесконечности и

$$
l^{\prime}(t)=o\left(l(t) t^{-1}\right) \quad(t \rightarrow \infty) .
$$

Тогда $f(t) \sim g(t)$ при $t \rightarrow \infty$. Аналогичное следствие справедливо и относительно теоремь 2.

Отметим, что для произвольной медленно меняющейся функции сушествует эквивалентная ей на бесконечности функция $l(t)$, для которой справедливо (6). Это следует из теоремы о представлении для медленно меняющихся функций $[13$, с. 10].

Преобразование Лапласа функции $a(t)$ будем обозначать через $\widehat{a}(\lambda)$ :

$$
\widehat{a}(\lambda)=\int_{0}^{\infty} e^{-\lambda t} a(t) d t, \quad \lambda>0 .
$$

Доказательство теорем 1, 2 опирается на следуюшую тауберову теорему.

Теорема 3. Пусть функции $a(t)$ u $b(t)$ измеримы $и$ неотрицательны при $t \geqslant 0$, при $\lambda>0$ у них существуют преобразования Лапласа $\widehat{a}(\lambda)$ u $\widehat{b}(\lambda)$, функиия $b(t)$ для некоторого $\alpha \geqslant 0$ не возрастает при $t \geqslant \alpha$ и мажорируемо меняется на бесконечности:

$$
\varlimsup_{t \rightarrow \infty} \frac{b(t)}{b(2 t)}<\infty
$$

причем при $y \geqslant x, y=x+o(x)$

$$
\varlimsup_{x \rightarrow \infty} \frac{a(y)-a(x)}{b(x)} \leqslant 0 .
$$

Если существует $M \in \mathbf{N}$ такое, что для произвольного фиксированного $n \geqslant M$ при $\lambda \rightarrow 0$

$$
\frac{d^{n}}{d \lambda^{n}} \widehat{a}(\lambda)=(1+o(1)) \frac{d^{n}}{d \lambda^{n}} \widehat{b}(\lambda),
$$

mo

$$
a(t) \stackrel{\text { cл. }}{\sim} b(t) \quad(t \rightarrow \infty) .
$$


Первоначально докажем теорему 3 , а затем выведем из нее теоремы 1,2 .

Д ок а за те ль с т в о т е о ре мы 3. Так как функция $b(t)$ мажорируемо меняется на бесконечности (выполнено (7)), то по теореме п. 2 из [13] существует такое $M_{1} \geqslant M$, что для любого фиксированного $n \geqslant M_{1}$

$$
\varlimsup_{t \rightarrow \infty} \frac{\int_{0}^{t} u^{n} b(u) d u}{t^{n+1} b(t)}<\infty .
$$

Отсюда следует, что для таких $n$ и произвольного $\lambda \in(0,1)$

$$
\varlimsup_{t \rightarrow \infty} \frac{B_{n}(\lambda t)}{B_{n}(t)}<1
$$

где $B_{n}(t)=\int_{0}^{t} u^{n} b(u) d u$. В самом деле, (12) эквивалентно соотношению

$$
\varliminf_{t \rightarrow \infty} \frac{\int_{\lambda t}^{t} u^{n} b(u) d u}{\int_{0}^{t} u^{n} b(u) d u}>0
$$

а из (11) в силу невозрастания $b(t)$ следует (13). Далее, согласно лемме 1 из [11], функция $B_{n}(t)$ слабо осциллирует на бесконечности (см. определение 2). Поэтому из теоремы 11 работы [12] и соотношения (9) следует, что для произвольного фиксированного $n \geqslant M$

$$
A_{n}(t)=(1+o(1)) B_{n}(t) \quad(t \rightarrow \infty)
$$

где $A_{n}(t)=\int_{0}^{t} u^{n} a(u) d u$. Зафиксируем произвольное $\lambda \in(0,1)$. Согласно (12), существуют такие $t_{0}, c_{0}>0$, что при $t \geqslant t_{0}$

$$
\begin{aligned}
\left|\frac{A_{n}(t)-A_{n}(\lambda t)}{B_{n}(t)-B_{n}(\lambda t)}-1\right| & \leqslant c_{0}\left|\frac{A_{n}(t)-B_{n}(t)+B_{n}(\lambda t)-A_{n}(\lambda t)}{B_{n}(t)}\right| \\
& \leqslant c_{0}\left|\frac{A_{n}(t)}{B_{n}(t)}-1\right|+c_{0}\left|\frac{A_{n}(\lambda t)}{B_{n}(\lambda t)}-1\right| \frac{B_{n}(\lambda t)}{B_{n}(t)} .
\end{aligned}
$$

Последнее выражение есть $o(1)$ при $t \rightarrow \infty$ в силу (14). Стало быть, для произвольного фиксированного $\lambda \in(0,1)$ при $t \rightarrow \infty$

$$
\int_{\lambda t}^{t} u^{n} a(u) d u=(1+o(1)) \int_{\lambda t}^{t} u^{n} b(u) d u .
$$

Зафиксируем произвольные $\varepsilon, \delta \in(0,1)$. Согласно (7), существуют такие $x_{0} \geqslant 2 \alpha$ и $c>0$, что при $x \geqslant x_{0}$

$$
b(x) \leqslant c b(2 x)
$$

Положим

$$
\varepsilon_{1}=\min \left(\frac{\varepsilon}{2}, \frac{\varepsilon}{1+c}\right)
$$


Из (8) следует, что найдутся такие $\delta_{1} \in(0, \delta)$ и $x_{1} \geqslant x_{0}$, что для произвольных $x \geqslant x_{1}$ и $y \in\left[x, x\left(1+\delta_{1}\right)\right]$ выполнено неравенство

$$
a(y)-a(x) \leqslant \varepsilon_{1} b(x) .
$$

Из (15) следует, что при $x \rightarrow \infty$

$$
\int_{x}^{x\left(1+\delta_{1}\right)} u^{n} a(u) d u=(1+o(1)) \int_{x}^{x\left(1+\delta_{1}\right)} u^{n} b(u) d u .
$$

Согласно (19), найдется такое $x_{2} \geqslant x_{1}$, что для произвольных $x \geqslant x_{2}$

$$
\int_{x}^{x\left(1+\delta_{1}\right)} y^{n} a(y) d y \geqslant\left(1-\varepsilon_{1}\right) \int_{x}^{x\left(1+\delta_{1}\right)} y^{n} b(y) d y .
$$

Из (18) и (20) следует, что при $x \geqslant x_{2}$

$$
\begin{aligned}
\int_{x}^{x\left(1+\delta_{1}\right)}\left(a(x)+\varepsilon_{1} b(x)\right) y^{n} d y & \geqslant \int_{x}^{x\left(1+\delta_{1}\right)} y^{n} a(y) d y \\
& \geqslant\left(1-\varepsilon_{1}\right) \int_{x}^{x\left(1+\delta_{1}\right)} y^{n} b(y) d y
\end{aligned}
$$

или же

$$
\begin{aligned}
& \left(a(x)+\varepsilon_{1} b(x)\right) x^{n+1} \frac{\left(1+\delta_{1}\right)^{n+1}-1}{n+1} \\
& \geqslant\left(1-\varepsilon_{1}\right) x^{n+1} \frac{\left(1+\delta_{1}\right)^{n+1}-1}{n+1} b\left(x\left(1+\delta_{1}\right)\right),
\end{aligned}
$$

откуда $a(x)+\varepsilon_{1} b(x) \geqslant b\left(x\left(1+\delta_{1}\right)\right)\left(1-\varepsilon_{1}\right)$. В силу (16) и монотонности $b(x)$ из последнего неравенства следует, что при $x \geqslant x_{2}$

$$
a(x)+c \varepsilon_{1} b\left(x\left(1+\delta_{1}\right)\right) \geqslant a(x)+\varepsilon_{1} b(x) \geqslant\left(1-\varepsilon_{1}\right) b\left(x\left(1+\delta_{1}\right)\right),
$$

откуда

$$
a(x) \geqslant\left(1-\varepsilon_{1}\right) b\left(x\left(1+\delta_{1}\right)\right)-c \varepsilon_{1} b\left(x\left(1+\delta_{1}\right)\right),
$$

или же

$$
a(x) \geqslant\left(1-(c+1) \varepsilon_{1}\right) b\left(x\left(1+\delta_{1}\right)\right), \quad x \geqslant x_{2} .
$$

Согласно (8), сушествуют $\delta_{2} \in(0, \delta), \delta_{2} \leqslant \frac{1}{2}$, и $x_{3} \geqslant x_{2}$ такие, что для произвольных $y \geqslant x_{3}$ и $x \in\left[y, y\left(1-\delta_{2}\right)^{-1}\right]$

$$
a(x)-a(y) \leqslant \varepsilon_{1} b(y)
$$

или же

$$
a(y) \geqslant a(x)-\varepsilon_{1} b(y) \geqslant a(x)-\varepsilon_{1} b\left(x\left(1-\delta_{2}\right)\right) .
$$

В силу (15) существует $x_{4} \geqslant x_{3}$ такое, что при $x \geqslant x_{4}$

$$
\int_{x\left(1-\delta_{2}\right)}^{x} y^{n} a(y) d y \leqslant\left(1+\varepsilon_{1}\right) \int_{x\left(1-\delta_{2}\right)}^{x} y^{n} b(y) d y .
$$


Из (22) и (23) получаем, что при $x \geqslant x_{4}$

$$
\begin{aligned}
\int_{x\left(1-\delta_{2}\right)}^{x} y^{n}\left(a(x)-\varepsilon_{1} b\left(x\left(1-\delta_{2}\right)\right)\right) d y & \leqslant \int_{x\left(1-\delta_{2}\right)}^{x} y^{n} a(y) d y \\
& \leqslant\left(1+\varepsilon_{1}\right) \int_{x\left(1-\delta_{2}\right)}^{x} y^{n} b(y) d y
\end{aligned}
$$

откуда в силу монотонности $b(x)$

$$
a(x)-\varepsilon_{1} b\left(x\left(1-\delta_{2}\right)\right) \leqslant\left(1+\varepsilon_{1}\right) b\left(x\left(1-\delta_{2}\right)\right),
$$

или же

$$
a(x) \leqslant\left(1+2 \varepsilon_{1}\right) b\left(x\left(1-\delta_{2}\right)\right),
$$

при $x \geqslant x_{4}$. Из соотношений $(17),(21)$ и (24) следует, что при $x \geqslant x_{4}$

$$
(1-\varepsilon) b\left(x\left(1+\delta_{1}\right)\right) \leqslant a(x) \leqslant(1+\varepsilon) b\left(x\left(1-\delta_{2}\right)\right) .
$$

Положим $x_{5}=\max \left(x_{4}, \alpha /(1-\delta)\right)$. В силу монотонности $b(x)$ из $(25)$ следует, что при $x \geqslant x_{5}$

$$
(1-\varepsilon) b(x(1+\delta)) \leqslant a(x) \leqslant(1+\varepsilon) b(x(1-\delta)) .
$$

Теорема 3 доказана.

Используя теорему 3, докажем теперь теорему 1.

Д ок а зательст в о те о ремы 1. Предположим первоначально, что $G((-\infty, 0))=0$. Точно так же, как и при доказательстве леммы 2 из работы [10], убеждаемся в том, что $F(t)$ дифференцируема при $t>0$, причем выполнено соотношение

$$
t f(t)=\int_{0}^{t}(t-y) g(t-y) d F(y)
$$

для всех $t>0$. При $\lambda \geqslant 0$ положим $\widetilde{F}(\lambda)=\int_{0}^{\infty} e^{-\lambda t} d F(t)$. Мы имеем:

$$
\widetilde{F}(\lambda)=\exp (-r(0))+\hat{f}(\lambda)=\exp \left(-\int_{0}^{\infty}\left(1-e^{-\lambda t}\right) g(t) d t\right) .
$$

Дифференцируя (27) $n$ раз как сложную функцию, получаем, что

$$
\hat{f}^{(n)}(\lambda)=\tilde{F}(\lambda) \sum_{i_{1}, \ldots, i_{n}} C\left(i_{1}, \ldots, i_{n}\right) \prod_{j=1}^{n}\left(\widehat{g}^{(j)}(\lambda)\right)^{i_{j}}
$$

где суммирование ведется по всем упорядоченным наборам неотрицательных целых чисел $i_{1}, \ldots, i_{n}$, удовлетворяющих условию $\sum_{j=1}^{n} j i_{j}=n$, а $C\left(i_{1}, \ldots, i_{n}\right)$ - некоторые константы. Пусть $k \in \mathbf{N}$ таково, что

$$
I_{k}=\int_{0}^{\infty} t^{k} g(t) d t=\infty
$$


Возьмем произвольное $n \geqslant k$. Тогда, согласно лемме 7 из [5], для всех $i_{1}, \ldots, i_{n}$ таких, что $i_{n}=0$, мы имеем:

$$
\prod_{j=1}^{n}\left(\widehat{g}^{(j)}(\lambda)\right)^{i_{j}}=o\left(\left|\widehat{g}^{(n)}(\lambda)\right|\right) \quad(\lambda \rightarrow 0) .
$$

Так как $C(0,0, \ldots, 0,1)=1$, то из $(28)$ с учетом (29) получаем, что

$$
\hat{f}^{(n)}(\lambda)=(1+o(1)) \widehat{g}^{(n)}(\lambda) \quad(\lambda \rightarrow 0) .
$$

Положим $a(t)=t f(t), b(t)=t g(t), t \geqslant 0$. Из (30) следует, что для всех $n \geqslant k-1$ выполнено соотношение (9) теоремы 3. Далее, соотношение (7) этой теоремы тривиальным образом следует из (1). Для того чтобы воспользоваться теоремой 3 , остается доказать, что выполнено (8). Займемся этим. В терминах функций $a(t)$ и $b(t)$ соотношение $(26)$ перепишется в виде

$$
a(t)=\int_{0}^{t} b(t-u) d F(u) .
$$

Из (31) следует, что при $x \geqslant \alpha$ и $y \geqslant x$

$$
\begin{aligned}
a(y)-a(x) & =\int_{0}^{x}(b(y-u)-b(x-u)) d F(u)+\int_{x}^{y} b(y-u) d F(u) \\
& \leqslant \int_{x-\alpha}^{x}(b(y-u)-b(x-u)) d F(u)+\int_{x}^{y} b(y-u) d F(u) .
\end{aligned}
$$

Поэтому

$$
a(y)-a(x) \leqslant \int_{x-\alpha}^{y} b(y-u) d F(u) \leqslant \sup _{v \geqslant 0} b(v) \cdot(T(x-\alpha)-T(y)),
$$

где $T(u)=1-F(u), u \geqslant 0$. Заметим, что при $y \geqslant x \geqslant \alpha$

$$
\begin{aligned}
0 & \leqslant \frac{r(x)-r(y)}{r(x)}=\frac{\int_{x}^{y} g(u) d u}{\int_{x}^{\infty} g(u) d u} \leqslant \frac{\int_{x}^{y} g(u) d u}{\int_{x}^{2 x} g(u) d u} \\
& \leqslant \frac{g(x)(y-x)}{x g(2 x)}=\left(\frac{y}{x}-1\right) \frac{g(x)}{g(2 x)} .
\end{aligned}
$$

Последнее выражение есть $o(1) \cdot O(1)=o(1)$ при $x \rightarrow \infty, y=x+o(x)$ в силу (1) и монотонности $g(x)$. Стало быть, $r(x)$ слабо осциллирует на бесконечности. Поэтому, воспользовавшись следствием 1 из работы [5], отсюда получаем, что

$$
T(x)=(1+o(1)) r(x) \quad(x \rightarrow \infty) .
$$

Заметим, что условие (2) равносильно соотношению

$$
\varliminf_{t \rightarrow \infty} \frac{\int_{t}^{\lambda t} g(x) d x}{\int_{t}^{\infty} g(x) d x}>0
$$


В свою очередь, (34) равносильно неравенству

$$
\varlimsup_{t \rightarrow \infty} \frac{r(t)}{\int_{t}^{\lambda t} g(x) d x}<\infty .
$$

А соотношение (35) в силу мажорируемой изменчивости функции $g(x)$ выполнено тогда и только тогда, когда

$$
\varlimsup_{t \rightarrow \infty} \frac{r(t)}{t g(t)}<\infty \text {. }
$$

Из (33) следует, что при $y \geqslant x, x \rightarrow \infty, y=x+o(x)$

$$
\begin{aligned}
T(x-\alpha)-T(y) & =(1+o(1)) r(y)-(1+o(1)) r(x-\alpha) \\
& =r(y)-r(x-\alpha)+o(r(x))=o(r(x))
\end{aligned}
$$

в силу слабой осцилляции $r(x)$, которая доказана ранее. Учитывая (36), отсюда получаем, что при $y \geqslant x, x \rightarrow \infty, y=x+o(x)$

$$
T(x-\alpha)-T(y)=o(x g(x)) .
$$

Из соотношений $(32)$ и (37) следует (8). Воспользовавшись тауберовой теоремой 3 , получаем, что $a(t) \stackrel{\text { сл. }}{\sim} b(t)(t \rightarrow \infty)$, откуда следует, что $f(t) \stackrel{\text { лл. }}{\sim} g(t)(t \rightarrow \infty)$. Таким образом, в рассмотренном частном случае теорема доказана. В случае, когда $\sigma=0, f(t)$ представляется в виде

$$
f(t)=\int_{0}^{\infty} p(t+x) d H(x), \quad t>0,
$$

где $H(x)$ - функция безгранично делимого распределения с мерой Леви $G_{1}(A)=G(-A \cap(-\infty, 0)), p(t)$ - производная функции безгранично делимого распределения с мерой Леви $G_{2}(A)=G(A \cap(0, \infty))(A-$ произвольное борелевское множество из $\left.\mathbf{R}^{1}\right)$. Положим

$$
f_{1}(t)=\int_{0}^{\sqrt{t}} p(t+x) d H(x), \quad t>0 .
$$

Покажем сначала, что

$$
f_{1} \stackrel{\text { сл. }}{\sim} g(t) \quad(t \rightarrow \infty)
$$

Зафиксируем произвольные $\varepsilon, \delta \in(0,1)$. Положим $\varepsilon_{1}=1-\sqrt{1-\varepsilon}, \delta_{1}=$ $\sqrt{1+\delta}-1$. Согласно доказанному, сушествует $t_{1} \geqslant \alpha /(1-\delta)$ такое, что $\left(1-\varepsilon_{1}\right) g\left(y\left(1+\delta_{1}\right)\right) \leqslant p(y) \leqslant\left(1+\varepsilon_{1}\right) g\left(y\left(1-\delta_{1}\right)\right)$ при $y \geqslant t_{1}$, откуда при $y \geqslant t_{1}$

$$
\left(1-\varepsilon_{1}\right) g\left(y\left(1+\delta_{1}\right)\right) \leqslant p(y) \leqslant(1+\varepsilon) g(y(1-\delta))
$$

так как $\varepsilon_{1}=1-\sqrt{1-\varepsilon}<\varepsilon, \delta_{1}=\sqrt{1+\delta}-1<\delta$. Отсюда при $x \geqslant 0$ и $t \geqslant t_{1}$

$$
p(t+x) \leqslant(1+\varepsilon) g((t+x)(1-\delta)) \leqslant(1+\varepsilon) g(t(1-\delta)) .
$$


Далее, при $t \geqslant t_{2}=\max \left(t_{1}, \delta_{1}^{-2}\right)$ и $x \leqslant \sqrt{t}$ будут выполнены неравенства

$$
\begin{aligned}
p(t+x) & \geqslant\left(1-\varepsilon_{1}\right) g\left((t+x)\left(1+\delta_{1}\right)\right) \geqslant\left(1-\varepsilon_{1}\right) g\left(t\left(1+t^{-1 / 2}\right)\left(1+\delta_{1}\right)\right) \\
& \geqslant\left(1-\varepsilon_{1}\right) g\left(t\left(1+\delta_{1}\right)^{2}\right)=\left(1-\varepsilon_{1}\right) g(t(1+\delta))
\end{aligned}
$$

в силу определения $\delta_{1}$. Найдется $t_{3} \geqslant t_{2}$ такое, что при $t \geqslant t_{3} H(\sqrt{t}) \geqslant$ $1-\varepsilon_{1}$. Поэтому в силу неравенств (41) и (42) мы имеем при $t \geqslant t_{3}$

$$
H(\sqrt{t})\left(1-\varepsilon_{1}\right) g(t(1+\delta)) \leqslant f_{1}(t) \leqslant(1+\varepsilon) g(t(1-\delta)),
$$

откуда $\left(1-\varepsilon_{1}\right)^{2} g(t(1+\delta)) \leqslant f_{1}(t) \leqslant(1+\varepsilon) g(t(1-\delta))$, или же, в силу определения $\varepsilon_{1}$,

$$
(1-\varepsilon) g(t(1+\delta)) \leqslant f_{1}(t) \leqslant(1+\varepsilon) g(t(1-\delta)) .
$$

Из (43) непосредственно следует (40). Заметим, что

$$
\int_{\sqrt{t}}^{\infty} p(t+x) d H(x) \leqslant \sup _{x \geqslant t} p(x) \cdot(1-H(\sqrt{t})) .
$$

Так как $p(t) \stackrel{\text { сл. }}{\sim} g(t)$, то отсюда следует, что

$$
\int_{\sqrt{t}}^{\infty} p(t+x) d H(x)=o(g(t)) \quad(t \rightarrow \infty)
$$

Из (38), (39) и (44) выводим, что

$$
f(t)=f_{1}(t)+o(g(t)) \quad(t \rightarrow \infty) .
$$

Из (40) и (45) следует, что $f(t) \stackrel{\text { cл. }}{\sim} g(t)$. В случае $\sigma \neq 0$ применяются точно такие же рассуждения, что и раньше. Теорема 1 доказана.

Доказательство теоремы 2 проводится аналогично теореме 1.

Сделаем еше одно заключительное замечание. Как хорошо известно из теории мажорируемо меняющихся функций [13], (1) выполнено тогда и только тогда, когда найдутся такие положительные $c, x_{0}$ и действительное $\beta$, что при $y \geqslant x \geqslant x_{0}$ выполнено неравенство

$$
\frac{g(y)}{g(x)} \leqslant c\left(\frac{y}{x}\right)^{\beta}
$$

Поэтому для того чтобы было выполнено (2), достаточно, чтобы в (46) $\beta<-1$. В самом деле, $(2)$ имеет место тогда и только тогда, когда

$$
\varliminf_{t \rightarrow \infty} \frac{\int_{t}^{\lambda t} g(x) d x}{\int_{t}^{\infty} g(x) d x}>0,
$$

что равносильно соотношению

$$
\varlimsup_{t \rightarrow \infty} \frac{\int_{t}^{\infty} g(x) d x}{\int_{t}^{\lambda t} g(x) d x}<\infty .
$$


Поскольку $g(x)$ мажорируемо меняется, то (48) выполнено тогда и только тогда, когда $\overline{\lim }_{t \rightarrow \infty} \int_{t}^{\infty} g(x) d x /(t g(t))<\infty$. Делая в последнем интеграле замену $x=u t$, приходим к соотношению

$$
\varlimsup_{t \rightarrow \infty} \int_{1}^{\infty} \frac{g(u t)}{g(t)} d u<\infty .
$$

Остается заметить, что (49), а стало быть, и (2), выполнено, если неравенство (46) справедливо при некотором $\beta<-1$.

В заключение я хотел бы выразить свою глубокую признательность рецензенту профессору Владимиру Михайловичу Золотареву, который является родоначальником исследуемого направления, за ценные замечания и пожелания. В частности, он отметил, что открытым является вопрос об асимптотике плотности распределения $F(x)$ в случае, когда $1-F(x)$ либо медленно меняется на бесконечности, либо стремится $\mathrm{k}$ нулю быстрее любой степенной функции.

\section{СПИСОК ЛИТЕРАТУРЫ}

1. Антонов С. Н., Круглов В. М. Об асимптотическом поведении безгранично делимых распределений в банаховом пространстве. - Теория вероятн. и ее примен., 1982 , т. 27, в. 4 , с. $625-642$.

2. Круглов В.М., Антонов С.Н. Еще раз об асимптотическом поведении безгранично делимых распределений в банаховом пространстве. - Теория вероятн. и ее примен., 1984 , т. 29 , в. 4 , с. $735-742$.

3. Золотарев В. М. Об асимптотическом поведении одного класса безгранично делимых законов распределения. - Теория вероятн. и ее примен., 1961, т. 6, в. 3 , c. 303-334.

4. Сеибнев M. С. Асимптотика безгранично делимых распределений в $\mathbf{R}$. - Сиб. матем. журн., 1990, т. 31, № 1, с. 135-140.

5. Якымив А.Л. Асимптотическое поведение одного класса безгранично делимых распределений. - Теория вероятн. и ее примен., 1987, т. 32, в. 4, с. 691-702.

6. Embrechts P., Goldie Ch. M., Veraverbeke N. Subexponentiality and infinite divisibility. - Z. Wahrscheinlichkeitstheor. verw. Geb., 1979, v. 49, p. 335-347.

7. Grübel R. Über unbegrenzt teilbare Verteilungen. - Arch. Math., 1983, v. 41, № 1, p. $80-88$.

8. Omey E. Infinite divisibility and random sums of random vectors. - Yokohama Math. J., 1985, v. 33, № 1-2, p. 39-48.

9. Золотарев В.M. Аналитическое строение безгранично делимых законов класса L. - Литов. матем. сб., 1963, т. 3, № 1, с. 123-139.

10. Якьцмив А.Л. Асимптотика плотности безгранично делимого распределения на бесконечности. - Проблемы устойчивости стохастических моделей. М.: ВНИИСИ, 1990, с. 123-131.

11. Якьлмив А.Л. Асимптотические свойства моментов изменения состояний в случайном процессе рекордов. - Теория вероятн. и ее примен., 1986, т. 31, в. 3 , c. $577-581$.

12. Якьмив А.Л. Асимптотика вероятности продолжения критических ветвящихся процессов Беллмана-Харриса. - Тр. МИАН СССР, 1986, т. 177, с. 177-205.

13. Сенета Е. Правильно меняющиеся функции. М.: Наука, 1985, 141 с.

Поступила в редакцию

10.IV.2000 